\title{
Computational Physics with Particles - Nonequilibrium Molecular Dynamics and Smooth Particle Applied Mechanics
}

\author{
Wm. G. Hoover \\ Ruby Valley Research Institute \\ Highway Contract 60, Box 598, Ruby Valley 89833, NV USA
}

(Rec. August 26, 2007)

\begin{abstract}
Microscopic and macroscopic particle-simulation methods can both be applied to interesting nonequilibrium problems. Here I develop and discuss the ordinary differential equations underlying these two approaches and illustrate them with applications of interest to statistical mechanics and computational fluid mechanics.
\end{abstract}

Key words: molecular dynamics, computational methods, smooth particles

PACS numbers: 02.70.ns, 45.10.-b, 46.15.-x, 47.11.Mn

\section{INTRODUCTION}

Materials in motion can be understood from two quite different points of view. The microscopic particle view underlies statistical mechanics and kinetic theory $[1,2]$. The macroscopic field-theory view underlies continuum mechanics and the finite-element and finite-difference approaches of computational fluid mechanics [3]. In the late 1950s I became fascinated with the possibilities of numerical simulation. At the National Laboratories Alder, Fermi, Vineyard, and Wood were pioneering computational approaches to numerical simulation. After I finished graduate school Berni Alder attracted me to the Lawrence Livermore National Laboratory and to the University of California's nearby Department of Appied Science at UCDavis/Livermore. I had been fortunate to attend graduate school at the University of Michigan at a time when all the tools necessary to simulation were available - Andy De Rocco's statistical mechanics course, George Uhlenbeck's kinetic theory course, a fast computer, "MAD", the Michigan Algorithmic Decoder, and a crash course (two hours in the evening, total) in FORTRAN. With this background it was natural for me to find a job in the National Laboratories, where molecular dynamics and computational fluid mechanics were undergoing rapid development.
In this introductory talk I concentrate on the underlying fundamentals and on the similarities linking the microscopic and macroscopic particle approaches.

Two doctoral students at UCDavis' Department of Applied Science made major contributions to this work (see Fig. 1). Bill Ashurst was interested in solving nonequilibrium problems with molecular dynamics. We developed nonequilibrium molecular dynamics together in the early 1970s. Twenty years later Oyeon Kum came to Livermore from Korea. We worked together on macroscopic "smooth particle" methods for solving the fieldtheory problems of continuum mechanics. The problems opened up through these two kinds of studies are the subject of this talk. I will say a bit about the underlying numerical methods and then illustrate both the microscopic and the macroscopic approaches applied to three problem types: expanding gases, collapsing liquids, and failing solids.

The basic ideas underlying the microscopic approach can be found in my book, Computational Statistical Mechanics, available free at my website, http://williamhoover.info while those underlying the macroscopic smooth-particle approach can be found in my book, Smooth Particle Applied Mechanics - the State of the Art, vol. 25 in World Scientific Publishers' Advanced Series in Nonlinear Dyna- 

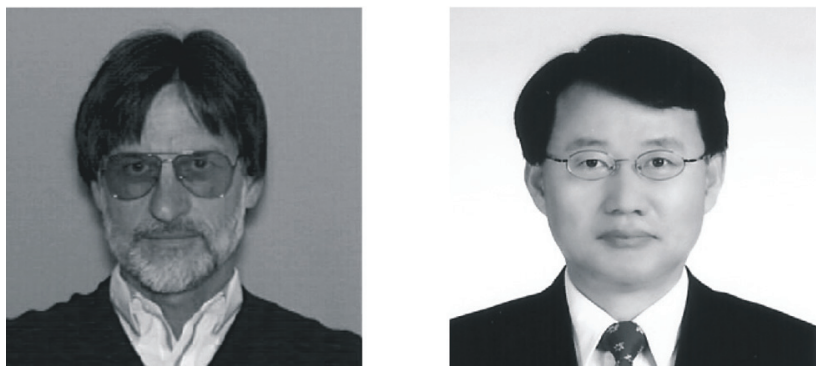

Fig. 1. William Ashurst and Oyeon Kum, pioneering research students in nonequilibrium molecular dynamics and smooth particle applied mechanics
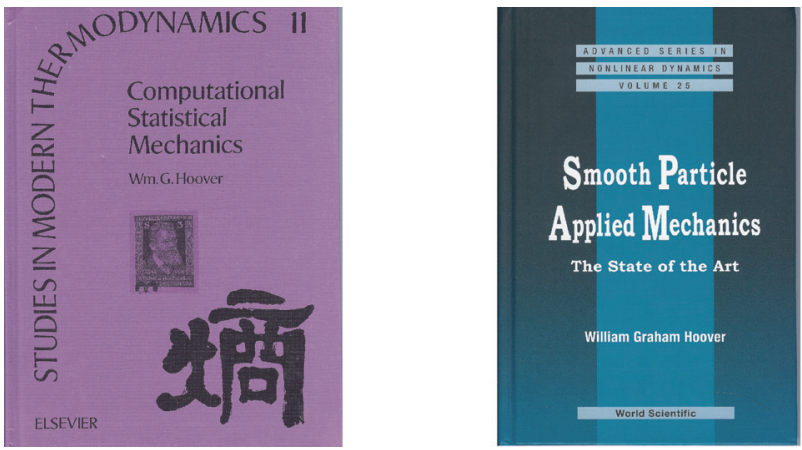

Fig. 2. Computational Statistical Mechanics and Smooth Particle Applied Mechanics, two useful references for the work described here

mics (see Fig. 2). It is worth stating at the outset that many users of smooth-particle methods call the method "sph", for Smooth Particle Hydrodynamics, a name which I have avoided because it suggests, wrongly, that the particle method is restricted to fluids. The method was discovered, simultaneously and independently, by two research groups working at Cambridge University $[4,5]$.

\section{NUMERICAL METHOD FOR ORDINARY DIFFERENTIAL EQUATIONS}

To follow the evolving state of a particulate system involves solving "motion equations". In microscopic molecular dynamics, these equations give the time derivatives $\{\dot{r}, \dot{v}\}$. of the coordinates and velocities in terms of the current system state $\{r, v\}$. A macroscopic particulate representation of continuum mechanics requires also an evolution equation for the energy $\{\dot{e}\}$ along with a more complicated state description, including at least energy, and perhaps additional derived variables, such as "plastic strain" and "damage".
Once the ordinary differential equations are formulated, solving them, even millions of them, is straightforward. A variety of numerical solution methods can be found in Numerical Recipes and other texts [6]. For simplicity, accuracy, and transparency I prefer the classic "fourth-order" Runge-Kutta method. Though this approach requires four evaluations of the righthandsides of the differential equations for each timestep $\Delta t$, the increased computer time is compensated by simplicity in programming and in controlling program execution.

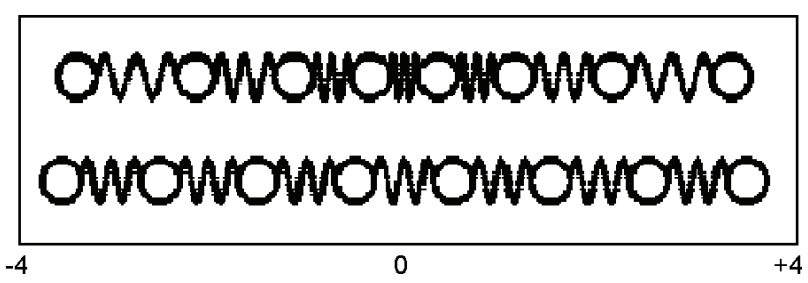

Fig. 3. An eight-particle harmonic chain, with displacements corresponding to its lowest-frequency oscillation

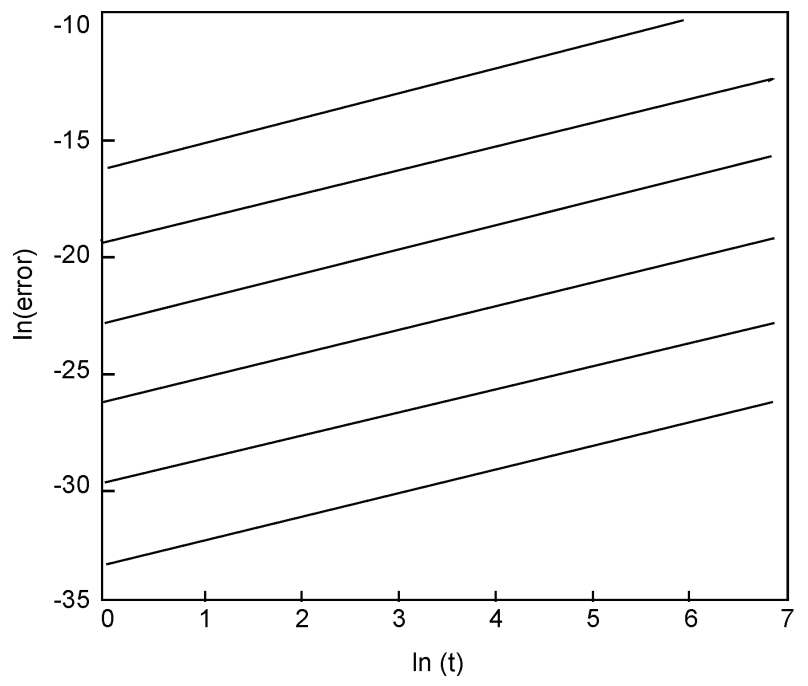

Fig. 4. Time dependence of the energy error in Runge-Kutta simulations of the chain shown in Fig. 3. The evolution of the error is shown for six timesteps, $\Delta t=\{0.01,0.02,0.04,0.08$, $0.16,0.32\}$. At a fixed time the error varies as the fifth power of the timestep $\Delta t$

To illustrate the Runge-Kutta method, consider the lowest-frequency oscillation of the eight-particle harmonic chain shown in Fig. 3. Figure 4 shows the time-dependence of the energy error for this chain problem. The RungeKutta method was used to solve the sixteen ordinary differential equations for the particles' coordinates and velocities: 


$$
\left\{\dot{x}_{i}=v_{i} ; \quad \dot{v}_{i}=x_{i-1}-2 x_{i}+x_{i+1}\right\} \text {. }
$$

Appropriate modifications were imposed at the ends ( $i=1$ and $i=8$ ), corresponding to imposing periodic (cyclic) boundary conditions on the chain. Figure 4 shows that the energy error at a fixed time $t$ (corresponding to $t / \Delta t$ timesteps) varies as the fifth power of the computational timestep $\Delta t$. Thus the smallest of the six timesteps used in constructing the Figure, $\Delta t=0.01$, reproduces the dynamics to double-precision accuracy. This same "fourth-order Runge-Kutta" algorithm is used in all of the examples I discuss here.

\section{FREE EXPANSION AND THE GIBBS-LIOUVILLE PARADOX}

The free expansion of a gas into a larger container is an interesting pedagogical problem. Because no work is done, and no heat is transferred, the energy is constant in such an expansion. Ideal gas thermodynamics gives an entropy increase of $N k \ln \left(V_{\text {Final }} / V_{\text {Initial }}\right)$ for this isoenergetic adiabatic process, where $k$ is Boltzmann's constant. Gibbs' and Boltzmann's statistical mechanics expresses entropy in terms of the averaged logarithm of the phase-space probability density $f(\{q, p\})$. The $\{p\}$ are the momenta conjugate to the generalized coordinates $\{q\}$ :

$$
\begin{gathered}
S_{\text {Gibbs }}=-k\langle\ln f\rangle \equiv \\
\equiv-k \prod_{i}\left[\iint d q_{i} d p_{i}\right] f \ln f .
\end{gathered}
$$

Liouville's Theorem, $\dot{f} \equiv 0$, is obeyed by a system of particles obeying Hamilton's motion equations,

$$
\{\dot{q}=+\partial \mathrm{H} / / \partial p ; / \quad \dot{p}=-\partial \mathrm{H} / / \partial q\} \rightarrow \dot{f} \equiv 0 .
$$

Liouville's Theorem, that $f$ (and hence also $\ln f$ ) is unchanged along the Hamiltonian trajectory, implies that Gibbs' entropy is likewise a constant of the motion. This constancy of entropy is paradoxical in light of the entropy increase predicted by thermodynamics.

Simulation gives insight into how this apparent paradox can be resolved. We simulate the expansion of a quiescent compressed gas obeying the ideal-gas equation of state, $P V=N k T=E$. Such a gas can be modeled accurately by using Lucy's normalized pair potential, shown in Fig. 5:

$$
\Phi=\sum_{i<j} \phi_{\text {Lucy }}\left(r_{i j}\right)
$$

$$
\begin{gathered}
\phi(r<h)_{\text {Lucy }}= \\
=\frac{5}{\pi h^{2}}\left[1-6(r / h)^{2}+8(r / h)^{3}-3(r / h)^{4}\right] . \\
\int_{0}^{\infty} \phi(r) 2 \pi r d r=\int_{0}^{h} \phi(r) 2 \pi r d r \equiv 1 \rightarrow E=\Phi \square \frac{N^{2}}{2 V} .
\end{gathered}
$$

The virial theorem for the pressure, along with an integration by parts, gives the desired dependence of the idealgas pressure on the (mostly potential) energy:

$$
\begin{gathered}
P V=\frac{1}{2} \sum_{i<j} r_{i j} \cdot F_{i j} \square-\frac{N^{2}}{4 V} \int_{0}^{h} \phi^{\prime} 2 \pi r^{2} d r=\frac{N^{2}}{4 V} \int_{0}^{h} \phi 4 \pi r d r= \\
=\Phi=\frac{N^{2}}{2 V}=E .
\end{gathered}
$$

Now imagine, as an initial condition, a checkerboard array of squares, one fourth of which are occupied by the compressed quiescent ideal gas (at a density $\rho=4$ ) with the remaining three-fourths of the squares empty. To compensate for the small missing "surface energy" of particles near the edge of the compressed square, the particles were given a small initial kinetic energy, just sufficient to provide a total energy $E=K+\Phi=2 N$ consistent with the bulk equation of state.

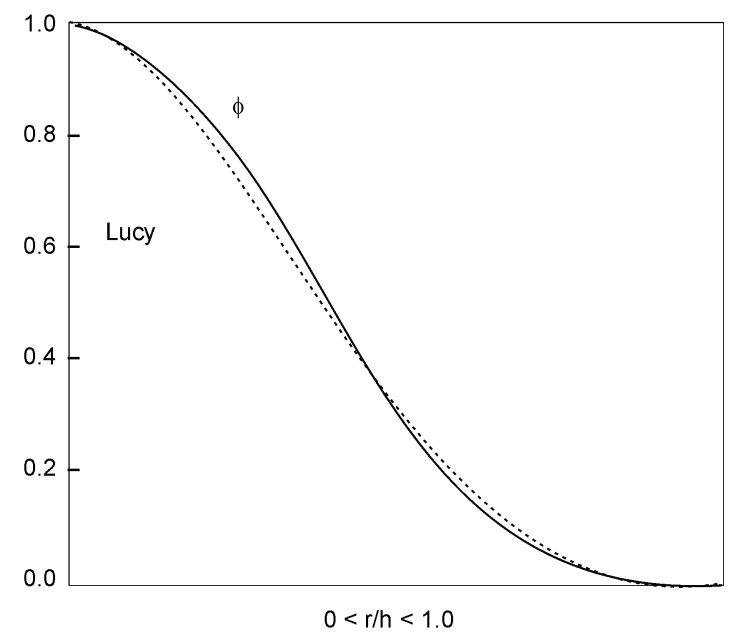

Fig. 5. Lucy's (unnormalized) weight function for $h=1$, $w(\mathrm{r}<1) \propto\left[1-6 r^{2}+8 r^{3}-3 r^{4}\right]$, is compared to the short-range repulsive potential, $\phi(r<1) \propto\left[1-r^{2}\right]^{4}$

To begin, the constraints confining the gas-filled square were released. Simulations, solving the $2 N$ motion equations, 

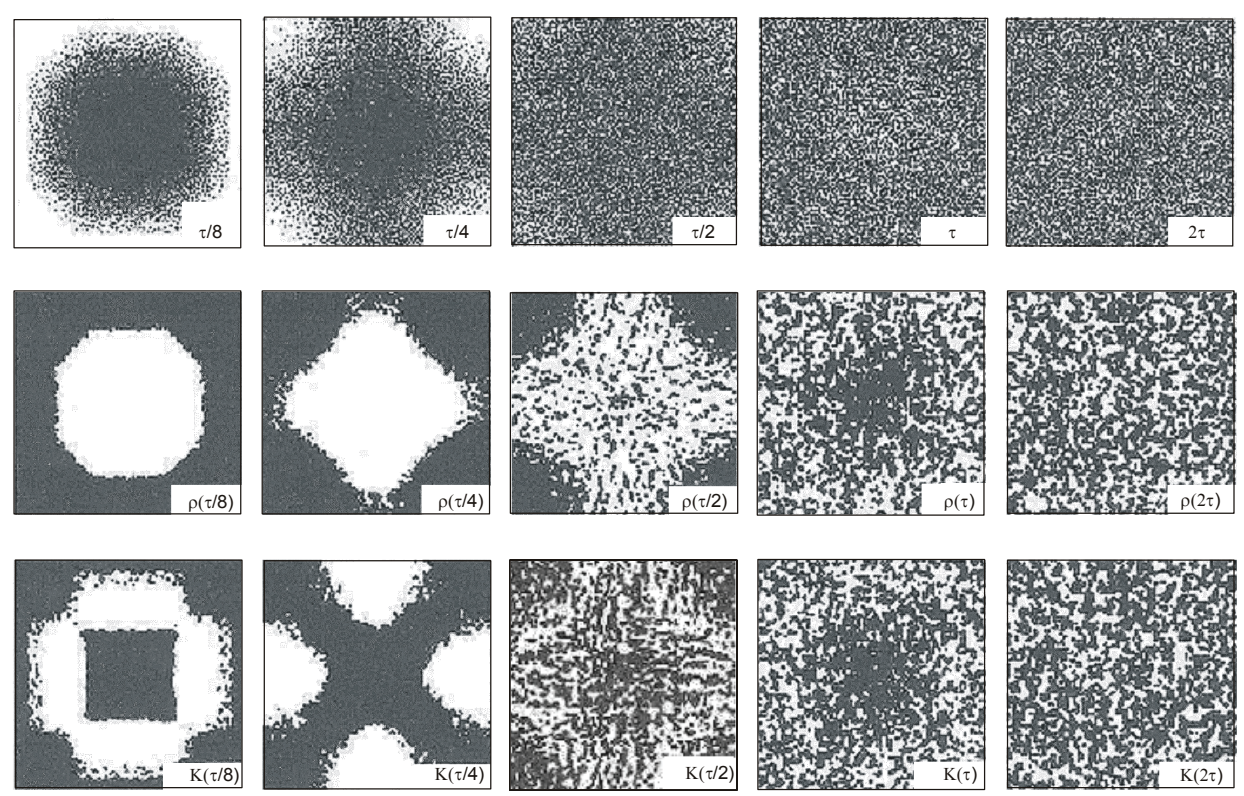

Fig. 6. Snapshots from a 16 384-particle free expansion (top row) in which the density decreases fourfold, from 4 to 1 . The average number of interacting neighbors varies from about $2 \pi h^{2} \square 60$ to $\pi h^{2} / 2 \square 15$ as the motion develops, where the range of the Lucy potential is $h=3$ and the particle mass is unity. The boundary separating the black and white regions is the contour of average density/kinetic energy in the two sets of contour plots. The total time interval shown corresponds to two sound-traversal times
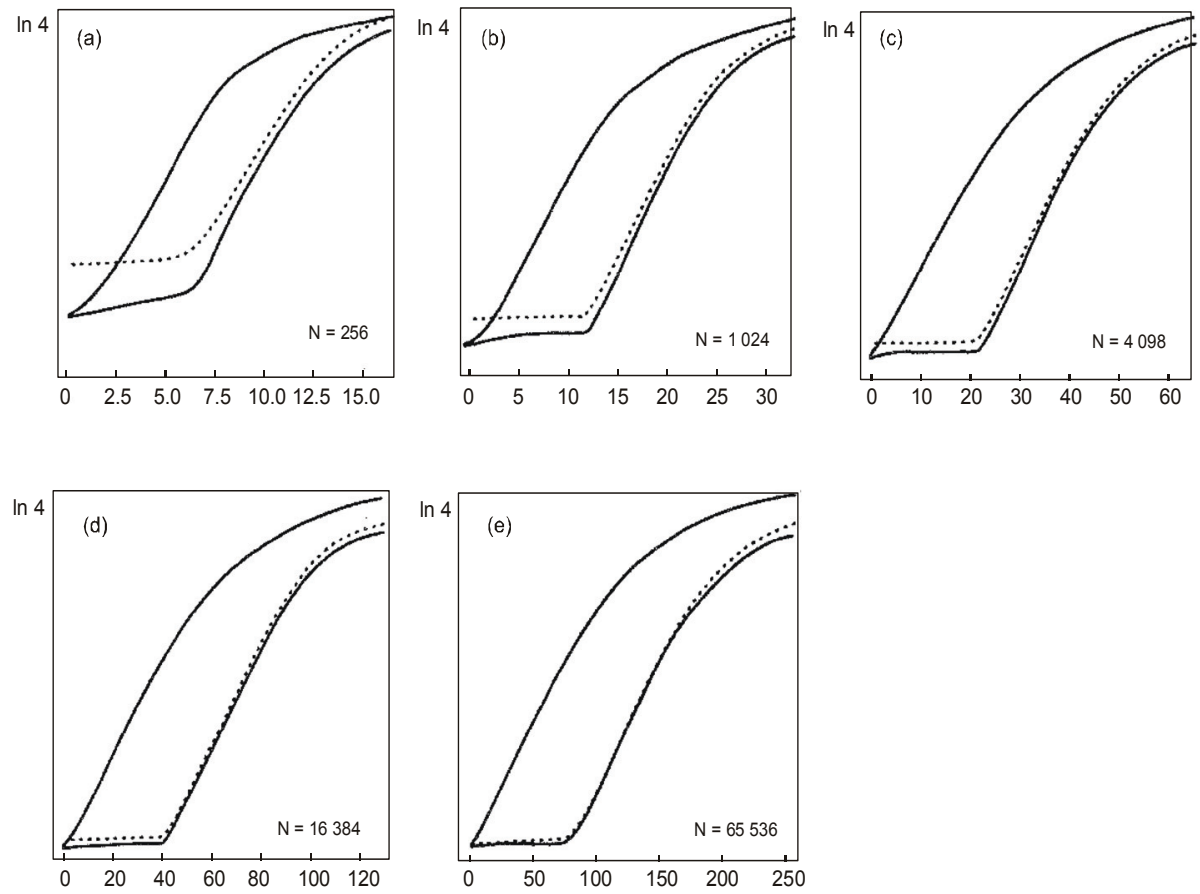

Fig. 7. Time dependence of the increase of entropy as a function of the number $N$ of smooth particles used in the free expansion problem. The entropy calculated here is based on the thermal energy fluctuation described above. The lower curve and the dots indicate particle-based and cell-based entropies. A third "entropy", based on the total thermal energy $K$ rather than its local fluctuation (the upper curves), wrongly indicates an entropy increase throughout the adiabatic expansion phase, even prior to the expanding fluid's impact with its periodic image (considered in Section IVC below) 


$$
\left\{\dot{r}_{i}=v_{i} ; m \dot{v}_{i}=\sum_{j} F_{i j}\right\},
$$

show that the subsequent relaxational motion equilibrates quickly, with the average density approaching $(\rho=1)$ in all the squares. Apart from the small surface corrections in the initial conditions, the density and energy changes are as follows:

$$
\begin{aligned}
& \rho: 4 \rightarrow 1 ; E / N: 2 \rightarrow 2 ; \\
& K / N: 0 \rightarrow 3 / 2 ; \frac{\Phi}{N}: 2 \rightarrow 1 / 2 .
\end{aligned}
$$

We model this problem here with molecular dynamics, using a "unit cell" of $2 \times 2=4$ squares, one full and three empty, and with periodic boundary conditions. Snapshots from a 16 384-particle simulation of this free expansion [7] are shown in Fig. 6.

In order to describe this expansion process from a macroscopic viewpoint it is useful to consider spatially-averaged values of the three field variables: density, velocity, and energy. Lucy's normalized potential is again useful for this averaging process, as it can play the role of a probability density (for a random distribution of particles). The average density at any point in space (such as the grid points on an interpolating mesh, for instance) can be defined as a sum of contributions from "nearby" (nearer than $h$ ) particles:

$$
\rho(r)=\sum_{i} m w\left(\left|r_{i}-r\right|<h\right) .
$$

The "weight function" $w$ describes the influence or contribution of a particle to the field variables in its neighborhood. In the same way, the spatially averaged velocity and kinetic energy $K$ can be defined:

$$
\begin{aligned}
& \rho(r) v(r)=\sum_{i} m w\left(\left|r_{i}-r\right|\right) v_{i} ; \\
& \rho(r) K(r)=\sum_{i} m w\left(\left|r_{i}-r\right|\right) \frac{m v_{i}^{2}}{2} .
\end{aligned}
$$

Figure 6 shows the division between those regions which have above-average and those which have below-average densities and kinetic energies. We see that two sound traversal times are enough for a nearly homogeneous distribution of mass, momentum, and energy to form throughout the system. Calculations of these field variables on a finely-meshed grid provide a precise description of the continuum evolution.
An understanding of the actual entropy increase of the expansion, shown in Fig. 7 for five different system sizes, despite Liouville's Theorem, can be based on the thermal energy fluctuation,

$$
\left\langle v^{2}\right\rangle-\langle v\rangle^{2} .
$$

The simple ideal-gas thermodynamic formula $S / N k=\ln (V T)$, where $T$ is the thermal energy, proportional to the velocity fluctuation, accounts nicely for the irreversible entropy increase associated with the free expansion. Introducing the weight function $w(r)$ provides both averages and fluctuations about the average values.

The averaging using $w(r)$ is a useful tool for a variety of problems. It can be applied to calculate the spatiallyaveraged value of any field function from the corresponding particle values:

$$
\begin{gathered}
F(r)=\sum_{j} w\left(\left|r_{j}-r\right|\right) F_{j} / \sum_{j} w\left(\left|r_{j}-r\right|\right)= \\
=\sum_{j} w_{j r} F_{j} / \sum_{j} w_{j r} .
\end{gathered}
$$

Because $w$ has two continuous spatial derivatives, with both vanishing at the range $h$ :

$$
(d w / d r)_{h}=0 ; \quad\left(d^{2} w / d r^{2}\right)_{h}=0,
$$

averages calculated in this way have two spatially-continuous derivatives everywhere. Such averages can be used to interpolate particle values onto a grid (for contour plots or Fourier transforms). They can also be used to create a particle representation, the "smooth particle" representation, of continuum mechanics, to which we turn in the next Section.

\section{SPAM: SMOOTH PARTICLE APPLIED MECHANICS}

The same averaging process used to describe the free expansion problem can be made the basis of a "smoothparticle" scheme to solve the field equations of continuum mechanics:

$$
\begin{gathered}
\dot{\rho}=-\rho \nabla \cdot v ; \quad \rho \dot{v}=-\nabla \cdot P ; \\
\rho \dot{e}=-\nabla \cdot Q-\nabla v: P .
\end{gathered}
$$

Here, the evolutions of the density, velocity, and internal energy $\{\rho(r, \mathrm{t}), v(r, t), e(r, t)\}$ depend upon both the pressure tensor $P$ and the heat flux vector $Q$. These two 
fluxes (of momentum and of energy) themselves depend upon past and present values of the field variables $\{\rho, v, e\}$. From the conceptual standpoint we imagine that particles, each of mass $\mathrm{m}$, have their own individual values,

$$
\left\{\rho_{i}, v_{i}, e_{i}, P_{i}, Q_{i},(\nabla v)_{i},(\nabla \cdot Q)_{i}, \ldots\right\}
$$

from which the corresponding field variables are computed as smooth-particle averages. Although there are ambiguities in choosing the basic definitions, these ambiguities provide opportunities, rather than drawbacks, in creating a useful numerical method for solving continuum problems. Here we next outline the smooth-particle representations of the continuity equation and the equation of motion.

\section{A. Smooth Particle Continuity Equation}

The continuity equation expresses conservation of mass. Equating the summed-up flows of mass into a fixed control volume to the density change in that volume leads directly to the continuity equation:

$$
\partial \rho / \partial t=-\nabla \cdot(\rho v) \longleftrightarrow \dot{\rho} \equiv \partial \rho / \partial t+v \cdot \nabla \rho=-\rho \nabla \cdot v
$$

The chain-rule time derivative of the smooth-particle representation of the density for Particle $i,(d / d t) \sum m w_{i j}$, gives a particle analog of the continuity equation:

$$
\begin{gathered}
\dot{\rho}_{i}=\sum m\left[v_{i} \cdot \nabla_{i} w_{i j}+v_{j} \cdot \nabla_{j} w_{i j}\right]= \\
=\sum m v_{i j} \cdot \nabla_{i} w_{i j}=\sum m\left(v_{i}-v_{j}\right) \cdot \nabla_{i} w_{i j}= \\
=v_{i} \cdot \nabla_{i} \rho_{i}-\nabla_{i} \cdot\left(\rho_{i} v_{i}\right)=-\rho_{i} \nabla_{i} \cdot v_{i} .
\end{gathered}
$$

The last equality follows from spatial differentiations of the two definitions:

$$
\begin{gathered}
\rho_{i}=\sum m w_{i j} \rightarrow v_{i} \nabla_{i} \rho=m v_{i} \cdot \nabla_{i} w_{i j} \\
(\rho v)_{i} \equiv \sum_{j} m v_{j} w_{i j} \rightarrow \nabla_{i}(p v)=\nabla_{i} m \sum_{j} v_{j} w_{i j} \equiv m \sum_{j} v_{j} \nabla_{i} w_{i j} .
\end{gathered}
$$

\section{B. Smooth Particle Motion Equation}

A force-balance calculation, equating the momentum change in a fixed volume element to the difference of the forces due to the pressure tensor $P$ gives the familiar continuum motion equation:

$$
\begin{gathered}
p \dot{v}=-\nabla \cdot P \longleftrightarrow \dot{v}= \\
=-\nabla \cdot(P / \rho)-\left(P / \rho^{2}\right) \cdot \nabla \rho .
\end{gathered}
$$

If we introduce smooth-particle definitions at Particle $i$ for these last two gradients:

$$
\begin{gathered}
\nabla \cdot(P / p) \rightarrow \sum_{j} m \nabla_{i} w_{i j}\left(P / \rho^{2}\right)_{j} ; \\
\nabla \rho \rightarrow \nabla_{i} \sum_{j} m w_{i j}=\sum_{j} m \nabla_{i} w_{i j},
\end{gathered}
$$

we see that the smooth-particle equation of motion can be written as a simple sum over pairs:

$$
\dot{v}_{i}=-m \sum_{j}\left[\left(\frac{P}{\rho^{2}}\right)_{i}+\left(\frac{P}{\rho^{2}}\right)_{j}\right] \cdot \nabla_{i} w_{i j} .
$$

This form of the motion equation, which follows from the two gradient definitions above, has the desirable feature that it conserves (linear) momentum exactly.

Whenever the pressure and density are slowly-varying in space the continuum equation of motion resembles molecular dynamics with a pair potential proportional to the weight function $w(r)$. In the two-dimensional ideal-gas case, with $P \propto \rho^{2}$, the resemblance becomes an isomorphism, with the molecular dynamics and SPAM particle trajectories identical.

\section{Fluid Interpenetration - Monaghan's Trick}

In continuum mechanics interfacial diffusion is ordinarily ignored so that adjacent fluids do not interpenetrate one another. In the usual smooth-particle case, with

$$
\begin{gathered}
\dot{r}_{i}=v_{i} \\
\dot{v}_{i}=-m \sum_{j}\left[\left(\frac{P}{\rho^{2}}\right)_{i}+\left(\frac{P}{\rho^{2}}\right)_{j}\right] \cdot \nabla_{i} w_{i j},
\end{gathered}
$$

the free-expansion example of the last Section leads to significant interpenetration. Monaghan introduced a clever variation of the evolution equations $\{\dot{r} \equiv v\}$ designed to minimize this effect while still conserving mass, momentum, and energy:

$$
\left\{\dot{r}_{i} \equiv v_{i}+m \sum_{j}\left(v_{j}-v_{i}\right) w_{i j} / \rho_{i j}\right\},
$$

where $\rho_{i j}$ is a mean density (either arithmetic or geometric). This motion based on the mean local velocity not only satisfies the conservation laws. It also prevents interpenetration. Figure 8 compares a conventional SPAM simulation of the free expansion (identical to a molecular dynamics simulation) with the improved and quite-different version incorporating Monaghan's modified equations of motion. 


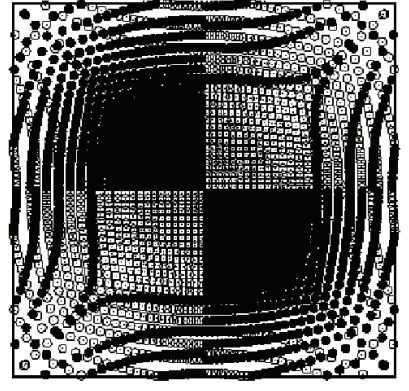

$-25<(x, y)<+25$

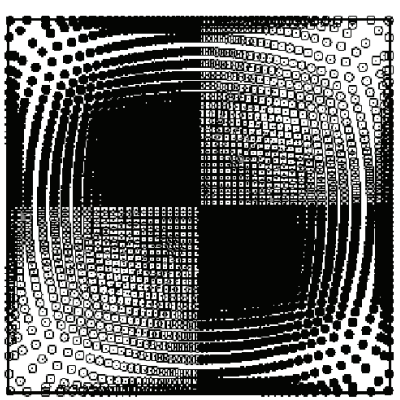

$-25<(x, y)<+25$
Fig. 8. Smooth-particle simulations of the expanding Lucy fluid. Particles initially located in the first and third quadrants are distinguished by shading. The conventional simulation (left) allows considerable interpenetration while that using Monaghan's Trick (right) does not

It would be useful to characterize how Monaghan's trick modifies hydrodynamic behavior. Because particles move according to an averaged neighborhood velocity rather than according to their own, we might expect reduced diffusion and enhanced viscosity to result. The equilibrium equation of state is not affected by Monaghan's trick. Homogeneous deformations do not activate the averaging. On the other hand, analysis of the dispersion relation $\omega(k)$ for solid-phase soundwaves shows enhanced dispersion and reduced oscillation frequencies for short wavelength waves.

\section{Smooth Particle Energy Equation}

In pairwise-additive molecular dynamics the natural definition of the single-particle energies is

$$
e_{i}=\frac{1}{2} \sum_{j} \phi_{i j}+\frac{m v_{i} \cdot v_{i}}{2} \text {. }
$$

The corresponding energy equation for molecular dynamics,

$$
\begin{gathered}
\dot{e}_{i}=\frac{1}{2} \sum \dot{\phi}_{i j}+m v_{i} \cdot \dot{v}_{i}= \\
=-\frac{1}{2} \sum\left(v_{i}-v_{j}\right) \cdot F_{i j}+\sum_{j} v_{i} \cdot F_{i j}= \\
=\frac{1}{2} \sum\left(v_{i}+v_{j}\right) \cdot F_{i j},
\end{gathered}
$$

turns out to be a simple identity, providing no new information. In continuum mechanics the energy equation includes both conductive and compressive energy changes, so that $e_{i}$ is an additional state variable. The smooth-particle version of the energy equation,

$$
\begin{aligned}
\dot{e}_{i}= & -\frac{m}{2} \sum\left[\left(\frac{P}{\rho^{2}}\right)_{i}+\left(\frac{P}{\rho^{2}}\right)_{j}\right]: v_{i j} \nabla_{i} w_{i j}+ \\
& -m \sum\left[\left(\frac{Q}{\rho^{2}}\right)_{i}+\left(\frac{Q}{\rho^{2}}\right)_{j}\right] \cdot \nabla_{i} w_{i j},
\end{aligned}
$$

conserves energy exactly, for either fluids or solids.

\section{E. SPAM vis-à-vis Molecular Dynamics}

We have emphasized the similarities between SPAM and molecular dynamics. In the general case the differences lie mainly in the accelerations. SPAM requires an a priori knowledge of the equation of state, including compressibility, heat capacity, transport coefficients, damage, and failure models. Molecular dynamics produces all these properties as functionals of the assumed interparticle force laws. SPAM, like real life, is ordinarily dissipative, with viscous forces tending to convert mechanical energy to heat. If it is desired to maintain thermal fluctuations, as in "Dissipative Particle Dynamics", this can be done with the same feedback forces used to control temperature in nonequilibrium molecular dynamics.

In addition to explicit viscosity and thermal feedback, the replacement of a continuum with a grid, such as the irregular grid formed by the SPAM particles, typically introduces a dissipative artificial viscosity. In the absence of any viscosity it is necessary to add in a von Neumann artificial viscosity to stabilize systems where shocks are formed. Molecular dynamics has none of these stability problems despite the characteristic Lyapunov instability of its motion equations.

The two techniques, though both involve particles, are best viewed as different descriptions of matter, macroscopic and microscopic respectively. Comparing the two descriptions for similar problems (Rayleigh-Bénard convection, shockwave formation, tensile collapse, penetration, ...) is an excellent approach to innovative research. Though failure (fracture, flow, fragmentation ...) is a universal phenomenon with intrinsic and economic motives for its study, much remains to be done in correlating the microscopic and macroscopic descriptions of failure mechanisms. In the space remaining I consider two examples of such problems, from both the macroscopic and the microscopic points of view. 


\section{TENSILE FAILURE WITH MOLECULAR DYNAMICS: GRAVITATIONAL EQUILIBRATION AND COLLAPSE OF A FLUID COLUMN}

Tensile failure can be initiated through expansion, either homogeneous or longitudinal, through the reflection of compressive waves from free boundaries ("spall"), or by the expansion of a compressed material. Quenching a "hot" compressed state to make a "cold" tensile state is yet another approach. One can hope that universal features of failure can be determined by comparing these processes with one another, all for the same material.

To illustrate tensile failure consider an equilibrated fluid column in a gravitational field $g$, constrained by a mirror boundary at its base and by lateral periodic boundaries. For simplicity we use a powerlaw mirror potential to support the column,

$$
\Phi_{\text {mirror }}=\sum_{i} 50 \delta y_{i}^{4} ; \quad \delta y=y_{i}<0 .
$$

We choose a short-ranged repulsive-attractive pair potential,

$$
\begin{gathered}
\phi(r<\sqrt{2})=\left[\left(2-r^{2}\right)^{8}-2\left(2-r^{2}\right)^{4}\right] \rightarrow \phi(0)=224 ; \\
\phi(1)=-1 .
\end{gathered}
$$

and constrain the kinetic energy per particle during the equilibration process, so as to avoid freezing:

$$
\left\langle m v^{2}\right\rangle=2 k T=1 .
$$

During the equilibration phase, this kinetic-energy constraint can be imposed by rescaling the particle velocities at the end of each Runge-Kutta timestep. A time of order several sound traversal times is ample for the finitetemperature equilibration used here, after which the lateral periodic boundary is released so that the column can expand laterally and collapse.

Figure 9 shows snapshots from a 5000-particle simulation, where the equilibrated height of the column (initially 100) is about 80 for a column width of 50 . The subsequent collapse generates a lateral expansion, which occurs at a speed somewhat less than the speed of sound. We can estimate the sound speed $c$ for a triangular lattice with the interparticle spacing and the particle mass both equal to unity and with a stress-free density of $\sqrt{4 / 3}$.

$$
c=\sqrt{(\partial P / \partial \rho)_{\rho=\sqrt{4 / 3}}} \square 9 .
$$

After the vertical boundary constraints are released, tensile "rarefaction waves" move inward from the newly-formed edges of the expanding column, eventually leading to sufficiently negative pressure to cause the formation of internal voids. The kinetics and morphology of the void formation is an interesting and challenging hydrodynamic subject area. Particular solution details depend on the type and the range of the interparticle forces. One could, for instance, explore the consequences of van der Waals'
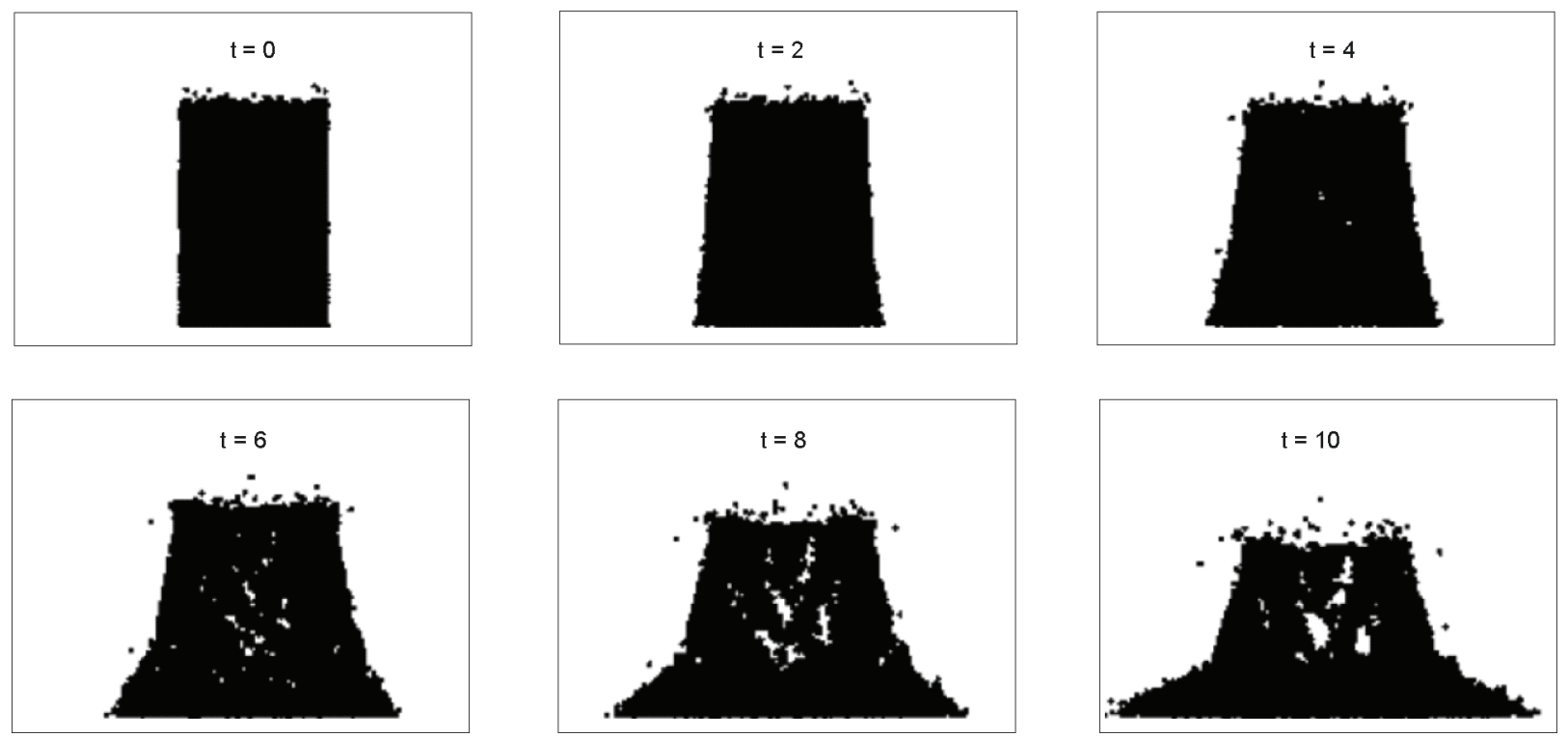

Fig. 9. Gravitational collapse of a pair-potential column. The equilibrated width is 50 and the total number of particles is 5000 . The strength of the gravitational field $g$ is 0.50 , as is also the thermal energy $k T$. A viscous relaxation time $\tau=10$ was applied for a time interval of 190 using a fourth-order Runge-Kutta timestep $\Delta t=0.01$. The time interval over which the collapse is illustrated here is for the subsequent time interval of 10 . Note the presence of tensile voids as well as some surface evaporation 
model by using a hard-core repulsive potential plus a longer-ranged attraction. A fundamental continuum treatment of the collapse process is also feasible. Such a treatment would involve formulating the dependence of the surface tension and viscosity on the local state variables, and the specification of a failure model leading to void formation. The irregular nature of the atomistic shape, for the system width shown here, of 50 atoms, certainly motivates the study of this same problem using continuum mechanics. We carry out such a simulation, with smooth continuum SPAM particles rather than atomistic ones, next.

\section{TENSILE FLUID FAILURE WITH SPAM: GRAVITATIONAL COLLAPSE OF A CONTINUUM COLUMN}

Now consider the continuum analog of the collapse problem, using SPAM. We choose a simple polynomial equation of state:

$$
P=(\rho / \bar{\rho})^{3}-(\rho / \bar{\rho})^{2}=\rho^{3}-\rho^{2},
$$

where $\bar{\rho}=1$ is the stress-free equilibrium density. For simplicity, we set both the particle mass and the stress-free density equal to unity here. The SPAM particle densities are calculated here as usual, using Lucy's form of the weighting function,

$$
\begin{gathered}
w(r<h)= \\
=\frac{5}{\pi h^{2}}\left[1-6(r / h)^{2}+8(r / h)^{3}-3(r / h)^{4}\right] .
\end{gathered}
$$

A more-nearly-correct simulation would include also a "failure model", which would describe the evolution of stress in the unstable regions of the equation of state. Though fluids can support tensile stresses of hundreds of atmospheres for long times, tension is thermodynamically unstable. Our equation of state is certainly mechanically unstable for densities less than $2 \bar{\rho} / 3$ where the compressibility becomes negative. A complete equation of state would have to include the possibility of a low-density vapor phase.

The smooth-particle equations of motion for our assumed equation of state take the form,
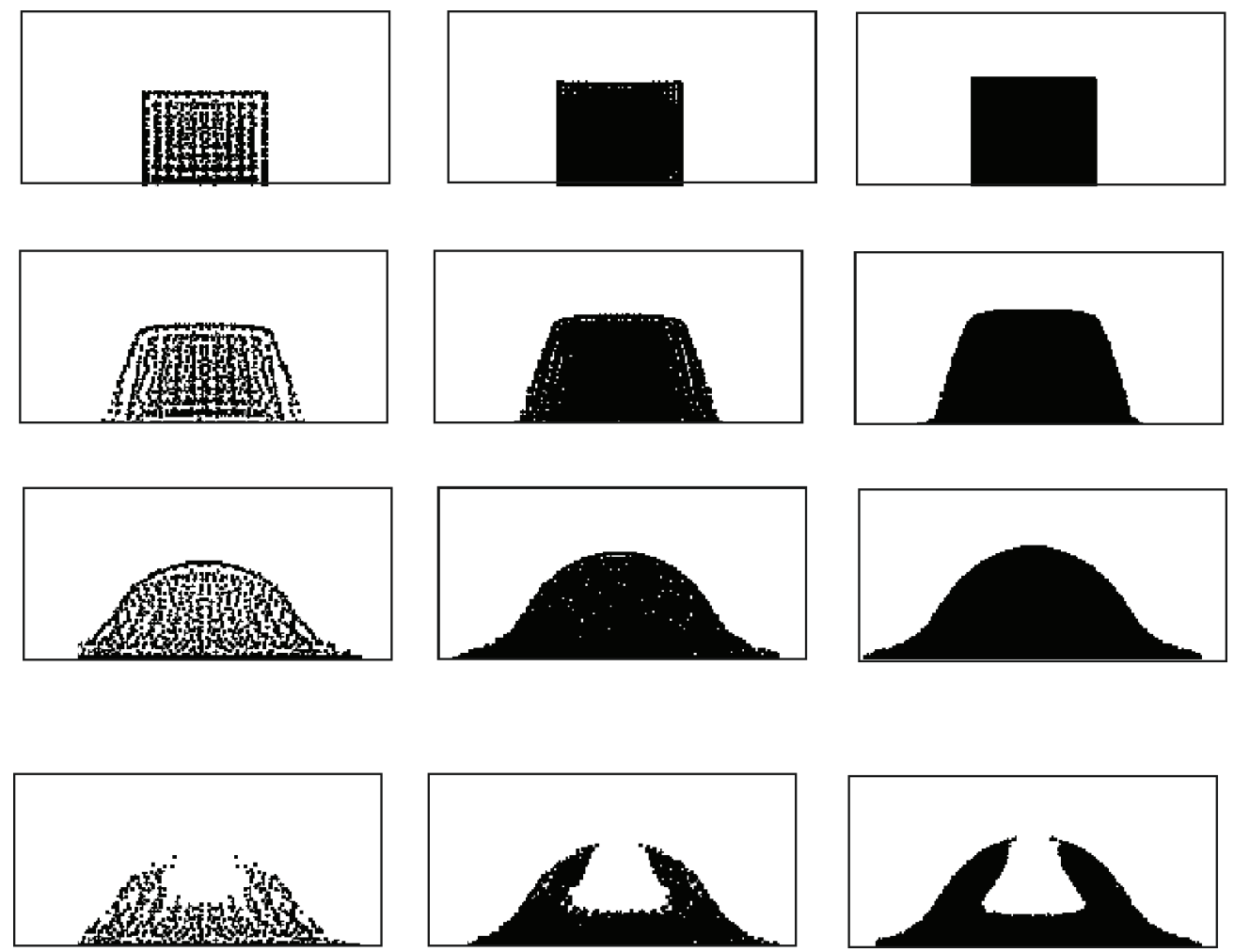

Fig. 10. Two successive stages of collapse of the initially-square equilibrated columns shown in the top row, as modeled by SPAM. Simulations with 640 and 2560 and 10240 particles are compared at corresponding times. The bottom row indicates the regions of positive pressure. These results are taken from Reference 3 


$$
\begin{gathered}
m \dot{v}_{i}=-\sum_{j}\left[\left(\rho_{i}-\bar{\rho}\right) \nabla_{i}\left(\rho_{i}\right)+\left(\rho_{j}-\bar{\rho}\right) \nabla_{i}\left(\rho_{j}\right)\right]= \\
=-\sum_{j}\left[\rho_{i}+\rho_{j}-2\right] \nabla_{i} w\left(r_{i j}\right) .
\end{gathered}
$$

Just as in molecular dynamics a Runge-Kutta solution of these motion equations conserves energy apart from a small single-step error, of order $\Delta t^{6}$. These SPAM motion equations are in fact equivalent to those computed in molecular dynamics from a manybody potential function designed to minimize density fluctuations:

$$
\Phi=\sum_{i} \phi_{i}(\rho)=\sum_{i} \frac{\left(\rho_{i}-1\right)^{2}}{2} .
$$

Although these SPAM motion equations do induce a density near unity for each particle, the model contains no intrinsic surface tension. Without surface tension the model tends to produce "strings" of particles. Though each particle has a density, $\sum m w$, close to the target density, the resulting "string-fluid" morphology is highly unrealistic. Surface tension can be added by including a phenomenological surface-energy potential designed to minimize density gradients,

$$
\Phi_{\text {surface }} \propto \sum_{i=1}^{N}\left(\nabla_{i} \rho\right)^{2} .
$$

Here we choose to use a surface potential with a proportionality constant of $1 / 10$.

Consider now the equilibration and collapse of a column of particles in a gravitational field $g$ induced by the gravitational potential $\Phi_{\text {grav }}=\sum_{i} m g y_{i}$. Figure 10 shows the tensile regions formed in the collapse of square equilibrated columns, as described by the smooth-particle motion equations. The good convergence, as indicated by the relative independence of the tensile-region shape to system size, indicates that a variety of failure models could be implemented so as to induce correspondence between the macroscopic SPAM and microscopic molecular dynamics simulations.

\section{PENETRATION}

Solids are intrinsically more complicated than fluids. At a minimum the elastic constants, yield strength, and a tensile failure model must be included for realistic studies. Figure 11 shows the progress of a round ball fired at an elastic-plastic plate. Atomistic simulations of such a problem are of relatively limited interest, because the defects (grain boundaries, dislocations, voids, impurities) responsible for the flow and failure properties require so many particles. Nevertheless, the multimillion atom simulations now underway can certainly suggest constitutive properties for inclusion in macroscopic models, such as SPAM, for such failure.
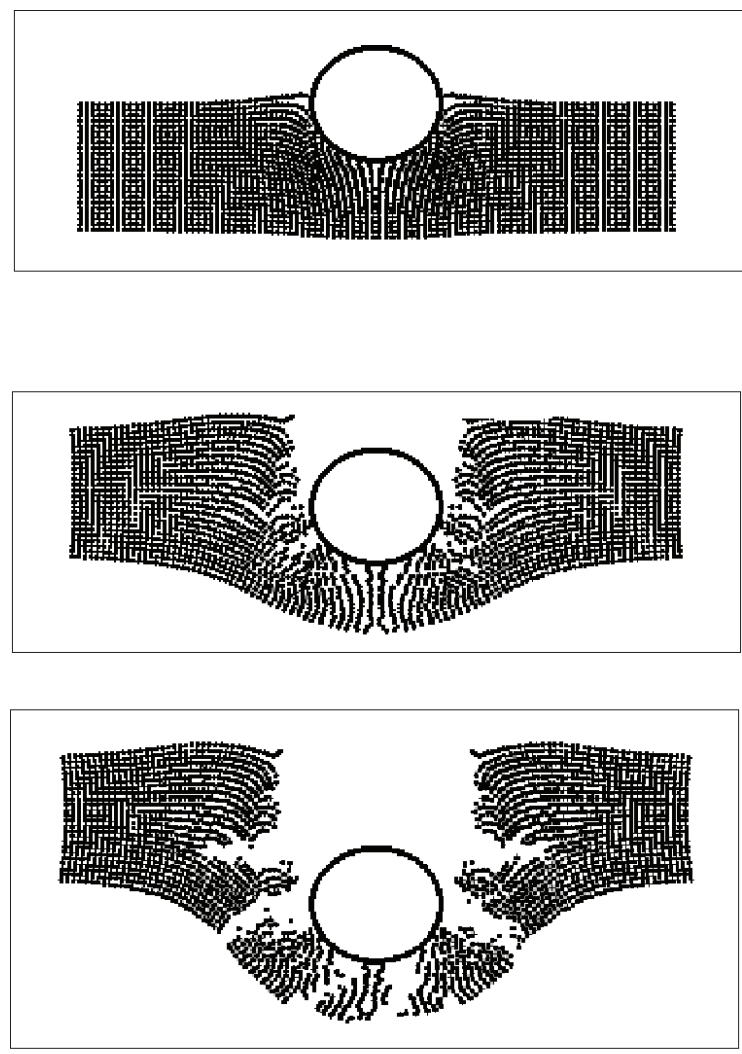

$$
-60<x<+60 ; \quad t=4,12,20
$$

Fig. 11. Penetration of a plate by a ball using smooth particles. The interaction between the (rigid) ball and the particles making up the plate was modeled by a purely-repulsive short-ranged pair potential. These results are taken from Reference 3

In treating such problems not only failure models, but also boundary conditions at material interfaces, are in need of developmental effort. The problem areas and solution techniques are mainly limited by imagination, now that the cost of highspeed computation is affordable.

\section{DISCUSSION}

I hope that this introduction to particle methods will motivate more research into nonequilibrium molecular dynamics and smooth particle applied mechanics. These complementary techniques offer detailed understanding of 
the mechanisms underlying constitutive behavior and provide a convenient means for modelling. Much remains to be done in discovering algorithms suitable to continuum boundary conditions, and models suited to describing failure. See, for example, Reference 8 . These areas are particularly suited to graduate-level computational research.

\section{Acknowledgment}

I thank Kris Wojciechowski and Billy Todd for suggesting and encouraging my work on this review. Karl Travis kindly read through the first draft of this manuscript and made several useful comments. My wife Carol helped me throughout.

\section{References}

[1] Wm. G. Hoover, Molecular Dynamics (Springer-Verlag, Berlin, 1986, available at the homepage http://williamhoover.info/MD.pdf).
[2] Wm. G. Hoover, Computational Statistical Mechanics (Elsevier, Amsterdam, 1991, available at the homepage http://williamhoover.info/book.pdf).

[3] Wm. G. Hoover, Smooth Particle Applied Mechanics - The State of the Art (World Scientific Publishers, Singapore, 2006, available from the publisher at the publisher's site http://www.worldscibooks.com/mathematics/6218.html).

[4] L. B. Lucy, A Numerical Approach to the Testing of the Fission Hypothesis, The Astronomical Journal 82, 1013 (1977).

[5] R. A. Gingold and J. J. Monaghan, Smoothed Particle Hydrodynamics: Theory and Application to Nonspherical Stars, Monthly Notices of the Royal Astronomical Society 181, 375-389 (1977).

[6] W. H. Press, B. P. Flannery, S. A. Teukolsky, and W. T. Vetterling, Numerical Recipes, the Art of Scientific Computing (Cambridge University Press, London, 1986).

[7] Wm. G. Hoover and H. A. Posch, Entropy Increase in Confined Free Expansions via Molecular Dynamics and Smooth Particle Applied Mechanics, Physical Review E 59, 1770-1776 (1999).

[8] O. Kum, Wm. G. Hoover and C. G. Hoover, Smooth-Particle Boundary Conditions, Physical Review E 68, 017701 (2003).

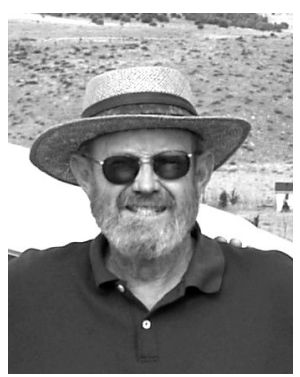

William Hoover got his graduate degrees at the University of Michigan and worked for more than forty years at the Lawrence Livermore National Laboratory and at the University of California's Davis campus. He is the author of about 300 papers in computational physics and has written four books on that subject. He and his wife Carol are now retired from the University of California and live in Ruby Valley, Nevada with their horses and cats. There the Hoovers also direct the Ruby Valley Research Institute. Their main research interests are nonequilibrium statistical mechanics and computational physics. Their main nonacademic pursuits are horseback riding and the planting of trees and flowers. 